\title{
Nonlocal boundary value problems for $\psi$-Hilfer fractional-order Langevin equations
}

\author{
Cholticha Nuchpong ${ }^{1}$, Sotiris K. Ntouyas ${ }^{2,3}$, Devaraj Vivek ${ }^{4}$ and Jessada Tariboon ${ }^{5^{*}}$ (D)
}

${ }^{*}$ Correspondence:

jessada.t@sci.kmutnb.ac.th

${ }^{5}$ Intelligent and Nonlinear Dynamic Innovations, Department of

Mathematics, Faculty of Applied

Science, King Mongkut's University

of Technology North Bangkok,

Bangkok, 10800, Thailand

Full list of author information is

available at the end of the article

\section{Springer}

\begin{abstract}
In the paper, we study a boundary value problem for a class of $\psi$-Hilfer fractional-order Langevin equations with multi-point integral boundary conditions. Existence and uniqueness results are established by using well-known fixed point theorems. Examples illustrating the main results are also included.
\end{abstract}

MSC: 26A33; 34A08; 34B10

Keywords: Boundary value problems; Langevin equations; $\psi$-Hilfer fractional derivative; Existence; Fixed point

\section{Introduction}

Fractional differential equations are important since their nonlocal property is suitable to characterize memory phenomena in economic, control, and material sciences. For the basic theory of fractional calculus and fractional differential equations we refer to [19]. Various types of fractional derivative and integral operator were studied: RiemannLiouville, Caputo, Hadamard, Erdelyi-Kober, Grünwald-Letnikov, Marchaud and Riesz are just a few examples. Hilfer [10] proposed a generalized Riemann-Liouville (R-L) fractional derivative, the so called Hilfer fractional derivative (HFD), which composites R-L fractional derivative and Caputo fractional derivative. HFD is performed, for example, in the theoretical simulation of dielectric relaxation in glass forming materials. Sousa and Oliveira [11] proposed the $\psi$-HFD and established $\psi$-Hilfer fractional differential equations. The fundamental discussion about existence and uniqueness of the solution of a nonlinear fractional-order differential equation involving $\psi$-HFD along with different types of initial conditions have been investigated in [12-18]. Asawasamrit et al. [19] studied the nonlocal boundary value problems for fractional-order differential equations with HFD subject to nonlocal integral boundary conditions. In [20], Saengthong et al. considered the existence results for Hilfer-Hadamard sequential fractional differential equations with two point boundary conditions. The study of a boundary value problem for fractional-order $\psi$-HFD was done by Harikrishnan et al. [21]. There has been published some significant work about the nonlinear boundary value problems for HFD, out of which we mention only a few that are relating to this article; see [22-24].

The note by Langevin [25] on Brownian motion had a permanent impact on scientific research and is still often cited. The Langevin equation is an effective tool of mathemati-

(c) The Author(s) 2021. This article is licensed under a Creative Commons Attribution 4.0 International License, which permits use, sharing, adaptation, distribution and reproduction in any medium or format, as long as you give appropriate credit to the original author(s) and the source, provide a link to the Creative Commons licence, and indicate if changes were made. The images or other third party material in this article are included in the article's Creative Commons licence, unless indicated otherwise in a credit line to the material. If material is not included in the article's Creative Commons licence and your intended use is not permitted by statutory regulation or exceeds the permitted use, you will need to obtain permission directly from the copyright holder. To view a copy of this licence, visit http://creativecommons.org/licenses/by/4.0/. 
cal physics, which can describe processes as regards time evolution of the velocity of the Brownian motion [26-28]. Among the applications of the Langevin equation, one can use modeling gait variability [29], financial markets [30] and described anomalous diffusion [31]. The existence and stability results for Langevin equations with HFD was considered in [32]. Several articles including a number of fractional derivatives have been considered by authors and researchers in the fractional Langevin equation, for instance [33-35]. Recently, three-point boundary value problems for Langevin equation with Hilfer fractional derivative were studied in [36].

In this paper, we consider a boundary value problem of Langevin fractional differential equations with $\psi$-HFD and nonlocal integral boundary conditions, given by

$$
\begin{aligned}
& \mathcal{D}^{\chi_{1}, \beta_{1} ; \psi}\left(\mathcal{D}^{\chi_{2}, \beta_{2} ; \psi}+k\right) x(t)=f(t, x(t)), \quad t \in J:=[a, b], \\
& x(a)=0, \quad x(b)=\sum_{i=1}^{m} \lambda_{i} I^{\delta_{i} ; \psi} x\left(\tau_{i}\right),
\end{aligned}
$$

where $\mathcal{D}^{\chi_{i}, \beta_{i} ; \psi}, i=1,2$ is the $\psi$-HFD of order $\chi_{i}, 0<\chi_{i}<1$ and type $\beta_{i}, 0 \leq \beta_{i} \leq 1, i=1,2$, $1<\chi_{1}+\chi_{2} \leq 2, k \in \mathbb{R}, a \geq 0, f: J \times \mathbb{R} \rightarrow \mathbb{R}$ is a continuous function, $I^{\delta_{i} i}$ is $\psi$-RiemannLiouville fractional integral of order $\delta_{i}>0, \lambda_{i} \in \mathbb{R}, i=1,2, \ldots, m$ and $0 \leq a \leq \tau_{1}<\tau_{2}<\cdots<$ $\tau_{m} \leq b$.

We prove two existence results by using Krasnosel'skii's fixed point theorem and the Leray-Schauder nonlinear alternative and one existence and uniqueness result via the Banach contraction mapping principle. The main results are presented in Sect. 3. Examples illustrating the main results are also constructed. In Sect. 2 we recall some preliminary facts which are needed in the following.

\section{Preliminaries}

We need some basic definitions and properties of fractional calculus that are used in this article. Let $\mathcal{C}=C(J, \mathbb{R})$ be the Banach space of all continuous functions from $J$ into $\mathbb{R}$ with the norm $\|x\|:=\sup \{|x(t)|: t \in J\}$.

Let $\psi \in C^{1}(J, \mathbb{R})$ be an increasing function with $\psi^{\prime}(t) \neq 0$ for all $t \in J$.

Definition 2.1 ([11]) Let $\chi>0(\chi \in \mathbb{R}), f \in L_{1}(J, \mathbb{R})$. Then the $\psi$-R-L fractional integral of a function $f$ with respect to $\psi$ is defined by

$$
I^{\chi ; \psi} f(t)=\frac{1}{\Gamma(\chi)} \int_{a}^{t} \psi^{\prime}(s)(\psi(t)-\psi(s))^{\chi-1} f(s) d s
$$

Definition 2.2 ([11]) Let $n-1<\chi<n \in \mathbb{N}$ and $f \in C^{n}(J, \mathbb{R})$. Then the $\psi$-HFD $\mathcal{D}^{\chi, \beta ; \psi}(\cdot)$ of a function $f$ of order $\chi$ and type $0 \leq \beta \leq 1$, is defined by

$$
\mathcal{D}^{\chi, \beta ; \psi} f(t)=I^{\beta(n-\chi) ; \psi}\left(\frac{1}{\psi^{\prime}(t)} \frac{d}{d t}\right)^{n} I^{(1-\beta)(n-\chi) ; \psi} f(t) .
$$

Lemma 2.1 ([11]) If $\in C^{n}[a, b], n-1<\chi<n$ and $0 \leq \beta \leq 1$, then

(1) $I^{\chi ; \psi} \mathcal{D}^{\chi, \beta ; \psi} f(t)=f(t)-\sum_{k=1}^{n} \frac{(\psi(t)-\psi(a))^{\gamma-k}}{\Gamma(\gamma-k+1)} f_{\psi}^{[n-k]} I^{(1-\beta)(n-\chi) ; \psi} f(a)$, 
where $f_{\psi}^{[n-k]} f(t)=\left(\frac{1}{\psi^{\prime}(t)} \frac{d}{d t}\right)^{n-k} f(t)$ and $\gamma=\chi+\beta(n-\chi)$.

(2) $\mathcal{D}^{\chi, \beta ; \psi} I^{\chi ; \psi} f(t)=f(t)$.

Next, we derive an equivalent fractional integral equation for a linear variant of the boundary value problem (1.1)-(1.2).

Lemma 2.2 Let $a \geq 0,0<\chi_{i}<1, \gamma_{i}=\chi_{i}+\beta_{i}\left(1-\chi_{i}\right), i=1,2,1<\chi_{1}+\chi_{2} \leq 2$, and $h \in$ $C([a, b], \mathbb{R})$. Then the function $x$ is a solution of the boundary value problem:

$$
\begin{aligned}
& \mathcal{D}^{x_{1}, \beta_{1} ; \psi}\left(\mathcal{D}^{\chi_{2}, \beta_{2} ; \psi}+k\right) x(t)=h(t), \\
& x(a)=0, \quad x(b)=\sum_{i=1}^{m} \lambda_{i} I^{\delta_{i} ; \psi} x\left(\tau_{i}\right),
\end{aligned}
$$

if and only if

$$
\begin{aligned}
x(t)= & I^{\chi_{1}+\chi_{2} ; \psi} h(t)-k I^{\chi_{2} ; \psi} x(t)+\frac{(\psi(t)-\psi(a))^{\gamma_{1}+\chi_{2}-1}}{\Lambda \Gamma\left(\gamma_{1}+\chi_{2}\right)}\left[\sum_{i=1}^{m} \lambda_{i} I^{\chi_{1}+\chi_{2}+\delta_{i} ; \psi} h\left(\tau_{i}\right)\right. \\
& \left.-k \sum_{i=1}^{m} \lambda_{i} I^{\chi_{2}+\delta_{i} ; \psi} x\left(\tau_{i}\right)-I^{\chi_{1}+\chi_{2} ; \psi} h(b)+k I^{\chi_{2} ; \psi} x(b)\right],
\end{aligned}
$$

where it is assumed that

$$
\Lambda=\frac{(\psi(b)-\psi(a))^{\gamma_{1}+\chi_{2}-1}}{\Gamma\left(\gamma_{1}+\chi_{2}\right)}-\sum_{i=1}^{m} \frac{\lambda_{i}}{\Gamma\left(\gamma_{1}+\chi_{2}+\delta_{i}\right)}\left(\psi\left(\tau_{i}\right)-\psi(a)\right)^{\gamma_{1}+\chi_{2}+\delta_{i}-1} \neq 0 .
$$

Proof Applying the $\psi$-R-L fractional integral of order $\chi_{1}$ to both sides of (2.1) and using Lemma 2.1 we obtain

$$
\mathcal{D}^{\chi_{2}, \beta_{2} ; \psi} x(t)+k x(t)=I^{\chi_{1} ; \psi} h(t)+\frac{c_{0}}{\Gamma\left(\gamma_{1}\right)}(\psi(t)-\psi(a))^{\gamma_{1}-1},
$$

where $c_{0}$ is an arbitrary constant and $\gamma_{1}=\chi_{1}+\beta_{1}\left(1-\chi_{1}\right)$.

Applying the $\psi$-R-L fractional integral of order $\chi_{2}$ to both sides of (2.4), we obtain

$$
\begin{aligned}
I^{\chi_{2} ; \psi} \mathcal{D}^{\chi_{2}, \beta_{2} ; \psi} x(t)= & I^{\chi_{1}+\chi_{2} ; \psi} h(t)-k I^{\chi_{2} ; \psi} x(t) \\
& +\frac{c_{0}}{\Gamma\left(\gamma_{1}+\chi_{2}\right)}(\psi(t)-\psi(a))^{\gamma_{1}+\chi_{2}-1} .
\end{aligned}
$$

Applying Lemma 2.1 to (2.5), we get

$$
\begin{aligned}
x(t)= & I^{\chi_{1}+\chi_{2} ; \psi} h(t)-k I^{\chi_{2} ; \psi} x(t)+\frac{c_{0}}{\Gamma\left(\gamma_{1}+\chi_{2}\right)}(\psi(t)-\psi(a))^{\gamma_{1}+\chi_{2}-1} \\
& +\frac{c_{1}}{\Gamma\left(\gamma_{2}\right)}(\psi(t)-\psi(a))^{\gamma_{2}-1} .
\end{aligned}
$$

Using $x(a)=0$ in (2.6), we obtain $c_{1}=0$, and hence we get

$$
x(t)=I^{\chi_{1}+\chi_{2} ; \psi} h(t)-k I^{\chi_{2} ; \psi} x(t)+\frac{c_{0}}{\Gamma\left(\gamma_{1}+\chi_{2}\right)}(\psi(t)-\psi(a))^{\gamma_{1}+\chi_{2}-1} .
$$


Next, by combining the second condition $x(b)=\sum_{i=1}^{m} \lambda_{i} I^{\delta_{i} ; \psi} x\left(\tau_{i}\right)$ of (2.2) with (2.7), we obtain

$$
c_{0}=\frac{1}{\Lambda}\left[\sum_{i=1}^{m} \lambda_{i} I^{\chi_{1}+\chi_{2}+\delta_{i} ; \psi} h\left(\tau_{i}\right)-k \sum_{i=1}^{m} \lambda_{i} I^{\chi_{2}+\delta_{i} ; \psi} x\left(\tau_{i}\right)-I^{\chi_{1}+\chi_{2} ; \psi} h(b)+k I^{\chi_{2} ; \psi} x(b)\right] .
$$

Substituting $c_{0}$ in (2.7) we get (2.3).

We can easily prove the converse by direct computation. The proof is completed.

Fixed point theorems play a major role in establishing the existence theory for the problem (1.1)-(1.2). We collect here some well-known fixed point theorems used in this paper.

Lemma 2.3 (Banach contraction principle [37]) Let D be a non-empty closed subset of a Banach space E. Then any contraction mapping $T$ from $D$ into itself has a unique fixed point.

Lemma 2.4 (Krasnosel'skii's fixed point theorem [38]) Let $\mathcal{M}$ be a closed, bounded, convex, and non-empty subset of a Banach space. Let $A, B$ be the operators such that (i) $A x+B y \in \mathcal{M}$ whenever $x, y \in \mathcal{M}$; (ii) $A$ is compact and continuous; (iii) $B$ is contraction mapping. Then there exists $z \in \mathcal{M}$ such that $z=A z+b z$.

Lemma 2.5 (Leray-Schauder nonlinear alternative [39]) Let E be a Banach space, $C$ a closed, convex subset of $E, U$ an open subset of $C$ and $0 \in U$. Suppose that $\mathcal{D}: \bar{U} \rightarrow C$ is a continuous, compact (that is, $\mathcal{D}(\bar{U})$ is a relatively compact subset of $C$ ) map. Then either

(i) $\mathcal{D}$ has a fixed point in $\bar{U}$, or

(ii) there is a $x \in \partial U$ (the boundary of $U$ in $C$ ) and $v \in(0,1)$ with $x=\nu \mathcal{D}(x)$.

\section{Main results}

In this section, we investigate the existence and uniqueness of solution for the boundary value problem (1.1)-(1.2).

By Lemma 2.2 we define an operator $\mathcal{N}: \mathcal{C} \rightarrow \mathcal{C}$ by

$$
\begin{aligned}
(\mathcal{N} x)(t)= & I^{\chi_{1}+\chi_{2} ; \psi} f(t, x(t))-k I^{\chi_{2} ; \psi} x(t) \\
& +\frac{(\psi(t)-\psi(a))^{\gamma_{1}+\chi_{2}-1}}{\Lambda \Gamma\left(\gamma_{1}+\chi_{2}\right)}\left[\sum_{i=1}^{m} \lambda_{i} I^{\chi_{1}+\lambda_{2}+\delta_{i} ; \psi} f\left(\tau_{i}, x\left(\tau_{i}\right)\right)\right. \\
& \left.-k \sum_{i=1}^{m} \lambda_{i} I^{\chi_{2}+\delta_{i} ; \psi} x\left(\tau_{i}\right)-I^{\chi_{1}+\chi_{2} ; \psi} f(b, x(b))+k I^{\chi_{2} ; \psi} x(b)\right], \quad t \in J .
\end{aligned}
$$

For the sake of brevity, we set

$$
\begin{aligned}
\Omega_{1}= & \frac{(\psi(b)-\psi(a))^{\chi_{1}+\chi_{2}}}{\Gamma\left(\chi_{1}+\chi_{2}+1\right)}+\frac{(\psi(b)-\psi(a))^{\gamma_{1}+\chi_{2}-1}}{|\Lambda| \Gamma\left(\gamma_{1}+\chi_{2}\right)} \\
& \times\left[\sum_{i=1}^{m}\left|\lambda_{i}\right| \frac{\left(\psi\left(\tau_{i}\right)-\psi(a)\right)^{\chi_{1}+\chi_{2}+\delta_{i}}}{\Gamma\left(\chi_{1}+\chi_{2}+\delta_{i}+1\right)}+\frac{(\psi(b)-\psi(a))^{\chi_{1}+\chi_{2}}}{\Gamma\left(\chi_{1}+\chi_{2}+1\right)}\right]
\end{aligned}
$$


and

$$
\begin{aligned}
\Omega_{2}=\mid & k \mid\left\{\frac{(\psi(b)-\psi(a))^{\chi_{2}}}{\Gamma\left(\chi_{2}+1\right)}+\frac{(\psi(b)-\psi(a))^{\gamma_{1}+\chi_{2}-1}}{|\Lambda| \Gamma\left(\gamma_{1}+\chi_{2}\right)}\right. \\
\times & {\left.\left[\sum_{i=1}^{m}\left|\lambda_{i}\right| \frac{\left(\psi\left(\tau_{i}\right)-\psi(a)\right)^{\chi_{2}+\delta_{i}}}{\Gamma\left(\chi_{2}+\delta_{i}+1\right)}+\frac{(\psi(b)-\psi(a))^{\chi_{2}}}{\Gamma\left(\chi_{2}+1\right)}\right]\right\} . }
\end{aligned}
$$

\subsection{Existence results}

We prove our first existence result for the boundary value problem (1.1)-(1.2) by using Krasnosel'skii's fixed point theorem [38].

\section{Theorem 3.1 Assume that:}

(H1) $f: J \times \mathbb{R} \rightarrow \mathbb{R}$ is a continuous function such that $|f(t, x(t))| \leq \varphi(t), \forall(t, x) \in J \times \mathbb{R}$, with $\varphi \in C(J, \mathbb{R})$.

(H2) $\Omega_{2}<1$, where $\Omega_{2}$ is given by (3.3).

Then there exists at least one solution for the boundary value problem (1.1)-(1.2) on J.

Proof We will show that the operator $\mathcal{N}$ defined by (3.1) satisfies the assumptions of Krasnosel'skii's fixed point theorem. We split the operator $\mathcal{N}$ into the sum of two operators $\mathcal{N}_{1}$ and $\mathcal{N}_{2}$ on the closed ball $\mathcal{B}_{\rho}=\{x \in C:\|x\| \leq \rho\}$ with $\rho \geq\left(\|\varphi\| \Omega_{1}\right) /\left(1-\Omega_{2}\right)$, $\sup _{t \in J} \varphi(t)=\|\varphi\|$, where

$$
\begin{aligned}
\left(\mathcal{N}_{1} x\right)(t)= & I^{\chi_{1}+\chi_{2} ; \psi} f(t, x(t))+\frac{(\psi(t)-\psi(a))^{\gamma_{1}+\chi_{2}-1}}{\Lambda \Gamma\left(\gamma_{1}+\chi_{2}\right)} \\
& \times\left[\sum_{i=1}^{m} \lambda_{i} I^{\chi_{1}+\chi_{2}+\delta_{i} ; \psi} f\left(\tau_{i}, x\left(\tau_{i}\right)\right)-I^{\chi_{1}+\chi_{2} ; \psi} f(b, x(b))\right], \quad t \in J,
\end{aligned}
$$

and

$$
\begin{aligned}
\left(\mathcal{N}_{2} x\right)(t)= & -k I^{\chi_{2} ; \psi} x(t)+\frac{(\psi(t)-\psi(a))^{\gamma_{1}+\chi_{2}-1}}{\Lambda \Gamma\left(\gamma_{1}+\chi_{2}\right)} \\
& \times\left[-k \sum_{i=1}^{m} \lambda_{i} I^{\chi_{2}+\delta_{i} ; \psi} x\left(\tau_{i}\right)+k I^{\chi_{2} ; \psi} x(b)\right], \quad t \in J .
\end{aligned}
$$

For any $x, y \in \mathcal{B}_{\rho}$, we have

$$
\begin{aligned}
& \left|\left(\mathcal{N}_{1} x\right)(t)+\left(\mathcal{N}_{2} y\right)(t)\right| \\
& \leq \sup _{t \in J}\left\{I^{\chi_{1}+\chi_{2}}|f(t, x(t))|+|k| I^{\chi_{2} ; \psi}|y(t)|+\frac{(\psi(t)-\psi(a))^{\gamma_{1}+\chi_{2}-1}}{|\Lambda| \Gamma\left(\gamma_{1}+\chi_{2}\right)}\right. \\
& \quad \times\left[\sum_{i=1}^{m}\left|\lambda_{i}\right| I^{\chi_{1}+\lambda_{2}+\delta_{i} ; \psi}\left|f\left(\tau_{i}, x\left(\tau_{i}\right)\right)\right|+I^{\chi_{1}+\chi_{2} ; \psi}|f(b, x(b))|\right. \\
& \left.\left.+|k| \sum_{i=1}^{m}\left|\lambda_{i}\right| I^{\chi_{2}+\delta_{i} ; \psi}\left|y\left(\tau_{i}\right)\right|+|k| I^{\chi_{2} ; \psi}|y(b)|\right]\right\}
\end{aligned}
$$




$$
\begin{aligned}
\leq & \|\varphi\|\left\{\frac{(\psi(b)-\psi(a))^{\chi_{1}+\chi_{2}}}{\Gamma\left(\chi_{1}+\chi_{2}+1\right)}+\frac{(\psi(b)-\psi(a))^{\gamma_{1}+\chi_{2}-1}}{|\Lambda| \Gamma\left(\gamma_{1}+\chi_{2}\right)}\right. \\
& \left.\times\left[\sum_{i=1}^{m}\left|\lambda_{i}\right| \frac{\left(\psi\left(\tau_{i}\right)-\psi(a)\right)^{\chi_{1}+\chi_{2}+\delta_{i}}}{\Gamma\left(\chi_{1}+\chi_{2}+\delta_{i}+1\right)}+\frac{(\psi(b)-\psi(a))^{\chi_{1}+\chi_{2}}}{\Gamma\left(\chi_{1}+\chi_{2}+1\right)}\right]\right\} \\
& +\|y\||k|\left\{\frac{(\psi(b)-\psi(a))^{\chi_{2}}}{\Gamma\left(\chi_{2}+1\right)}+\frac{(\psi(b)-\psi(a))^{\gamma_{1}+\chi_{2}-1}}{|\Lambda| \Gamma\left(\gamma_{1}+\gamma_{2}\right)}\right. \\
& \left.\times\left[\sum_{i=1}^{m}\left|\lambda_{i}\right| \frac{\left(\psi\left(\tau_{i}\right)-\psi(a)\right)^{\chi_{2}+\delta_{i}}}{\Gamma\left(\chi_{2}+\delta_{i}+1\right)}+\frac{(\psi(b)-\psi(a))^{\chi_{2}}}{\Gamma\left(\chi_{2}+1\right)}\right]\right\} \\
\leq & \|\varphi\| \Omega_{1}+\rho \Omega_{2} \leq \rho,
\end{aligned}
$$

and hence $\left\|\mathcal{N}_{1} x+\mathcal{N}_{2} y\right\| \leq \rho$, which implies that $\mathcal{N}_{1} x+\mathcal{N}_{2} y \in \mathcal{B}_{\rho}$. By using (H2) it is easy to prove that $\mathcal{N}_{2}$ is a contraction mapping.

The operator $\mathcal{N}_{1}$ is continuous, since $f$ is continuous. It is uniformly bounded on $\mathcal{B}_{\rho}$ as

$$
\left\|\mathcal{N}_{1} x\right\| \leq \Omega_{1}\|\varphi\| .
$$

Now, we prove that the operator $\mathcal{N}_{1}$ is compact. Setting $\sup _{(t, x) \in J \times \mathcal{B}_{\rho}}|f(t, x)|=\bar{f}<\infty$, we obtain

$$
\begin{aligned}
&\left|\left(\mathcal{N}_{1} x\right)\left(t_{2}\right)-\left(\mathcal{N}_{2} x\right)\left(t_{1}\right)\right| \\
& \leq \frac{\bar{f}}{\Gamma\left(\chi_{1}+\chi_{2}\right)} \mid \int_{a}^{t_{1}}\left[\psi^{\prime}(s)\left(\psi\left(t_{2}\right)-\psi(s)\right)^{\chi_{1}+\chi_{2}-1}-\psi^{\prime}(s)\left(\psi\left(t_{1}\right)-\psi(s)\right)^{\chi_{1}+\chi_{2}-1}\right] d s \\
&+\int_{t_{1}}^{t_{2}} \psi^{\prime}(s)\left(\psi\left(t_{2}\right)-\psi(s)\right)^{\chi_{1}+\chi_{2}-1} d s \mid \\
&+\frac{\left(\psi\left(t_{2}\right)-\psi(a)\right)^{\gamma_{1}+\chi_{2}-1}-\left(\psi\left(t_{1}\right)-\psi(a)\right)^{\gamma_{1}+\chi_{2}-1}}{|\Lambda| \Gamma\left(\gamma_{1}+\chi_{2}\right)} \\
& \times\left[\sum_{i=1}^{m}\left|\lambda_{i}\right| \bar{f} \frac{\left(\psi\left(\tau_{i}\right)-\psi(a)\right)^{\chi_{1}+\chi_{2}+\delta_{i}}}{\Gamma\left(\chi_{1}+\chi_{2}+\delta_{i}+1\right)}+\frac{(\psi(b)-\psi(a))^{\chi_{1}+\chi_{2}}}{\Gamma\left(\chi_{1}+\chi_{2}+1\right)}\right] \\
& \leq \frac{\bar{f}}{\Gamma\left(\chi_{1}+\chi_{2}+1\right)}\left[2\left(\psi\left(t_{2}\right)-\psi\left(t_{1}\right)\right)^{\chi_{1}+\chi_{2}}\right. \\
&\left.+\left|\left(\psi\left(t_{2}\right)-\psi(a)\right)^{\chi_{1}+\chi_{2}}-\left(\psi\left(t_{1}\right)-\psi(a)\right)^{\chi_{1}+\chi_{2}}\right|\right] \\
&+\frac{\left(\psi\left(t_{2}\right)-\psi(a)\right)^{\gamma_{1}+\chi_{2}-1}-\left(\psi\left(t_{1}\right)-\psi(a)\right)^{\gamma_{1}+\chi_{2}-1}}{|\Lambda| \Gamma\left(\gamma_{1}+\chi_{2}\right)} \\
& \times\left[\sum_{i=1}^{m}\left|\lambda_{i}\right| \bar{f} \frac{\left(\psi\left(\tau_{i}\right)-\psi(a)\right)^{\chi_{1}+\chi_{2}+\delta_{i}}}{\Gamma\left(\chi_{1}+\chi_{2}+\delta_{i}+1\right)}+\frac{(\psi(b)-\psi(a))^{\chi_{1}+\chi_{2}}}{\Gamma\left(\chi_{1}+\chi_{2}+1\right)}\right],
\end{aligned}
$$

which tends to zero as $t_{2}-t_{1} \rightarrow 0$, independently of $x \in \mathcal{B}_{\rho}$. Thus, $\mathcal{N}_{1}$ is equicontinuous and hence $\mathcal{N}_{1}$ is relatively compact on $\mathcal{B}_{\rho}$. By the Arzelá-Ascoli theorem, $\mathcal{N}_{1}$ is compact on $\mathcal{B}_{\rho}$. It follows by Krasnosel'skii's fixed point theorem (Lemma 2.4) that the problem (1.1)-(1.2) has at least one solution on $J$. The proof is complete. 
Example 3.1 Consider the boundary value problem of the Langevin equation with the $\psi$-HFD of the form

$$
\begin{aligned}
& \mathcal{D}^{\frac{1}{2}, \frac{1}{3} ; e^{t}}\left(\mathcal{D}^{\frac{3}{4}, \frac{1}{2} ; e^{t}}+\frac{1}{12}\right) x(t)=\frac{2 e^{\frac{1}{2}-t}}{2 t+15}\left(\frac{|x(t)|}{1+|x(t)|}\right)+\frac{1}{3}, \quad t \in\left[\frac{1}{2}, \frac{5}{2}\right], \\
& x\left(\frac{1}{2}\right)=0, \quad x\left(\frac{5}{2}\right)=\frac{1}{3} I^{\frac{1}{2} ;} ; e^{t} x(1)+\frac{1}{2} I^{\frac{3}{2}} ; e^{t} x\left(\frac{3}{2}\right)+\frac{3}{5} I^{\frac{5}{2} ; e^{t}} x(2) .
\end{aligned}
$$

Here $\chi_{1}=1 / 2, \chi_{2}=3 / 4, \beta_{1}=1 / 3, \beta_{2}=1 / 2, k=1 / 12, a=1 / 2, b=5 / 2, m=3, \delta_{1}=1 / 2, \delta_{2}=$ $3 / 2, \delta_{3}=5 / 2, \tau_{1}=1, \tau_{2}=3 / 2, \tau_{3}=2, \lambda_{1}=1 / 3, \lambda_{2}=1 / 2, \lambda_{3}=3 / 5$ and $\psi(t)=e^{t}$. We see that $\chi_{1}+\chi_{2}=5 / 4 \in(1,2]$. Using the given data, we get $\gamma_{1}=2 / 3, \gamma_{2}=7 / 8,|\Lambda| \approx 17.47973707$, $\Omega_{1} \approx 24.38179472$ and $\Omega_{2} \approx 0.9594635717$.

Observe that $\Omega_{2}<1$ and we obtain

$$
|f(t, x)|=\left|\frac{2 e^{\frac{1}{2}-t}}{2 t+15}\left(\frac{|x|}{1+|x|}\right)+\frac{1}{3}\right| \leq \frac{2 e^{\frac{1}{2}-t}}{2 t+15}+\frac{1}{3}:=\phi(t) .
$$

Thus all the assumptions of Theorem 3.1 are satisfied. Therefore by its conclusion, the boundary value problem (3.7)-(3.8) has at least one solution on [1/2,5/2].

Our next existence result is based on the Leray-Schauder nonlinear alternative (Lemma 2.5).

Theorem 3.2 Suppose that (H2) holds. In addition we assume that:

(H3) $|f(t, x)| \leq p(t) \phi(|x|)$ for each $(t, x) \in J \times \mathbb{R}$ where $\phi:[0, \infty) \rightarrow(0, \infty)$ is a continuous nondecresing function and $p \in C\left(J, \mathbb{R}^{+}\right)$.

(H4) There exists a constant $\mathcal{M}>0$, such that

$$
\frac{\left(1-\Omega_{2}\right) \mathcal{M}}{\phi(\mathcal{M})\|p\| \Omega_{1}}>1
$$

where $\Omega_{1}, \Omega_{2}$ are given by (3.2) and (3.3), respectively.

Then there exists at least one solution for the boundary value problem (1.1)-(1.2) on J.

Proof We will prove that the operator $\mathcal{N}$ defined by (3.1) satisfies the hypothesis of the Leray-Schauder nonlinear alternative. For this purpose, we first establish that operator $\mathcal{N}$ maps bounded sets (balls) into a bounded set in $\mathcal{C}$. Let $\mathcal{B}_{r}=\{x \in \mathcal{C}:\|x\| \leq r\}$ be a bounded ball with radius $r$ in $\mathcal{C}$. Then, for $t \in J$, we have

$$
\begin{aligned}
|(\mathcal{N} x)(t)| \leq & \sup _{t \in J}\left\{I^{\chi_{1}+\chi_{2} ; \psi}|f(t, x(t))|+|k| I^{\chi_{2} ; \psi}|x(t)|\right. \\
& +\frac{(\psi(t)-\psi(a))^{\gamma_{1}+\chi_{2}-1}}{\Lambda \Gamma\left(\gamma_{1}+\chi_{2}\right)}\left[\sum_{i=1}^{m}\left|\lambda_{i}\right| I^{\chi_{1}+\chi_{2}+\delta_{i} ; \psi}\left|f\left(\tau_{i}, x\left(\tau_{i}\right)\right)\right|\right. \\
& \left.\left.+|k| \sum_{i=1}^{m}\left|\lambda_{i}\right| I^{\chi_{2}+\delta_{i} ; \psi}\left|x\left(\tau_{i}\right)\right|+I^{\chi_{1}+\chi_{2} ; \psi}|f(b, x(b))|+|k| I^{\chi_{2} ; \psi}|x(b)|\right]\right\} \\
\leq & \|p\| \phi(\|x\|)\left\{\frac{(\psi(b)-\psi(a))^{\chi_{1}+\chi_{2}}}{\Gamma\left(\chi_{1}+\chi_{2}+1\right)}+\frac{(\psi(b)-\psi(a))^{\gamma_{1}+\chi_{2}-1}}{|\Lambda| \Gamma\left(\gamma_{1}+\chi_{2}\right)}\right.
\end{aligned}
$$




$$
\begin{aligned}
& \left.\times\left[\sum_{i=1}^{m}\left|\lambda_{i}\right| \frac{\left(\psi\left(\tau_{i}\right)-\psi(a)\right)^{\chi_{1}+\chi_{2}+\delta_{i}}}{\Gamma\left(\chi_{1}+\chi_{2}+\delta_{i}+1\right)}+\frac{(\psi(b)-\psi(a))^{\chi_{1}+\chi_{2}}}{\Gamma\left(\chi_{1}+\chi_{2}+1\right)}\right]\right\} \\
& +\|x\||k|\left\{\frac{(\psi(b)-\psi(a))^{\chi_{2}}}{\Gamma\left(\chi_{2}+1\right)}+\frac{(\psi(b)-\psi(a))^{\gamma_{1}+\chi_{2}-1}}{|\Lambda| \Gamma\left(\gamma_{1}+\gamma_{2}\right)}\right. \\
& \left.\times\left[\sum_{i=1}^{m}\left|\lambda_{i}\right| \frac{\left(\psi\left(\tau_{i}\right)-\psi(a)\right)^{\chi_{2}+\delta_{i}}}{\Gamma\left(\chi_{2}+\delta_{i}+1\right)}+\frac{(\psi(b)-\psi(a))^{\chi_{2}}}{\Gamma\left(\chi_{2}+1\right)}\right]\right\} \\
& =\|p\| \phi(\|x\|) \Omega_{1}+\|x\| \Omega_{2},
\end{aligned}
$$

and consequently

$$
\|\mathcal{N} x\| \leq\|p\| \phi(r) \Omega_{1}+\Omega_{2} r .
$$

Next, we will show that $\mathcal{N}$ maps bounded sets into equicontinuous sets of $\mathcal{C}$. Let $t_{1}, t_{2} \in J$ with $t_{1}<t_{2}$ and $x \in \mathcal{B}_{r}$. Then we have

$$
\begin{aligned}
& \left|(\mathcal{N} x)\left(t_{2}\right)-(\mathcal{N} x)\left(t_{1}\right)\right| \\
& \leq \frac{\|p\| \psi(r)}{\Gamma\left(\chi_{1}+\chi_{2}+1\right)}\left[2\left(\psi\left(t_{2}\right)-\psi\left(t_{1}\right)\right)^{\chi_{1}+\chi_{2}}\right. \\
& \left.\quad+\left|\left(\psi\left(t_{2}\right)-\psi(a)\right)^{\chi_{1}+\chi_{2}}-\left(\psi\left(t_{1}\right)-\psi(a)\right)^{\chi_{1}+\chi_{2}}\right|\right] \\
& \quad+\frac{\left(\psi\left(t_{2}\right)-\psi(a)\right)^{\gamma_{1}+\chi_{2}-1}-\left(\psi\left(t_{1}\right)-\psi(a)\right)^{\gamma_{1}+\chi_{2}-1}}{|\Lambda| \Gamma\left(\gamma_{1}+\chi_{2}\right)} \\
& \quad \times\left[\sum_{i=1}^{m}\left|\lambda_{i}\right|\|p\| \psi(r) \frac{\left(\psi\left(\tau_{i}\right)-\psi(a)\right)^{\chi_{1}+\chi_{2}+\delta_{i}}}{\Gamma\left(\chi_{1}+\chi_{2}+\delta_{i}+1\right)}+\|p\| \psi(r) \frac{(\psi(b)-\psi(a))^{\chi_{1}+\chi_{2}}}{\Gamma\left(\chi_{1}+\chi_{2}+1\right)}\right] \\
& \quad+\frac{|k| r}{\Gamma\left(\chi_{2}+1\right)}\left(\psi\left(t_{2}\right)-\psi\left(t_{1}\right)\right)^{\chi_{2}}+\frac{\left(\psi\left(t_{2}\right)-\psi(a)\right)^{\gamma_{1}+\chi_{2}-1}-\left(\psi\left(t_{1}\right)-\psi(a)\right)^{\gamma_{1}+\chi_{2}-1}}{|\Lambda| \Gamma\left(\gamma_{1}+\chi_{2}\right)} \\
& \quad \times\left[\sum_{i=1}^{m}\left|\lambda_{i}\right| \frac{\left(\psi\left(\tau_{i}\right)-\psi(a)\right)^{\chi_{2}+\delta_{i}}}{\Gamma\left(\chi_{2}+\delta_{i}+1\right)}+\frac{(\psi(b)-\psi(a))^{\chi_{2}}}{\Gamma\left(\chi_{2}+1\right)}\right] r .
\end{aligned}
$$

As $t_{1} \rightarrow t_{2}$, the right hand side of the above inequality tends to zero independently of $x \in$ $\mathcal{B}_{r}$. The set $\mathcal{N} \mathcal{B}_{r}$ is equicontinuous. Thus, we have proved that $\mathcal{N} \mathcal{B}_{r}$ is relatively compact. By the Arzelá-Ascoli theorem, $\mathcal{N}$ is completely continuous.

Finally, we show that the set of all solutions to equations $x=\omega \mathcal{N} x$ is bounded for $\omega \in$ $(0,1)$. Following the computation in the first step, we obtain

$$
|x(t)| \leq \phi(\|x\|)\|p\| \Omega_{1}+\|x\| \Omega_{2}, \quad t \in[a, b],
$$

which yields

$$
\frac{\left(1-\Omega_{2}\right)\|x\|}{\phi(\|x\|)\|p\| \Omega_{1}} \leq 1 .
$$

According to (H4), there exists $\mathcal{M}>0$ satisfying $\|x\| \neq \mathcal{M}$. Introduce the set

$$
U=\{x \in C(J, \mathbb{R}):\|x\|<\mathcal{M}\},
$$


and notice that $\bar{U} \rightarrow C$ is continuous and completely continuous. From the choice of $U$ there is no $x \in \partial U$, such that $x=\omega \mathcal{N} x$ for some $\omega \in(0,1)$. As a result of the nonlinear alternative of the Leray-Schauder type (Theorem 2.5) we deduce that $\mathcal{N}$ has a fixed point $x \in \bar{U}$, which is a solution of the boundary value problem (1.1)-(1.2). This completes the proof.

Example 3.2 Consider the boundary value problem of the Langevin equation with the $\psi$-HFD of the form

$$
\begin{aligned}
& \mathcal{D}^{\frac{2}{3}, \frac{4}{5} ; \log t}\left(\mathcal{D}^{\frac{4}{5}, \frac{2}{3} ; \log t}+\frac{1}{18}\right) x(t) \\
& =\frac{3}{3 t+1049}\left(x^{2}(t)+\frac{1}{2} e^{-x^{2}(t)}+\frac{1}{2}\right), \quad t \in\left[\frac{1}{3}, \frac{5}{3}\right], \\
& x\left(\frac{1}{3}\right)=0, \quad x\left(\frac{5}{3}\right)=\frac{1}{5} I^{\frac{3}{7} ; \log t} x\left(\frac{2}{3}\right)+\frac{2}{5} I^{\frac{4}{7} ; \log t} x(1)+\frac{3}{5} I^{\frac{5}{7} ; \log t} x\left(\frac{4}{3}\right) .
\end{aligned}
$$

Here $\chi_{1}=2 / 3, \chi_{2}=4 / 5, \beta_{1}=4 / 5, \beta_{2}=2 / 3, k=1 / 18, a=1 / 3, b=5 / 3, m=3, \delta_{1}=3 / 7$, $\delta_{2}=4 / 7, \delta_{3}=5 / 7, \tau_{1}=2 / 3, \tau_{2}=1, \tau_{3}=4 / 3, \lambda_{1}=1 / 5, \lambda_{2}=2 / 5, \lambda_{3}=3 / 5$ and $\psi(t)=\log t$. Observe that $\chi_{1}+\chi_{2}=22 / 15 \in(1,2]$.

With these values we found $\gamma_{1}=\gamma_{2}=14 / 15,|\Lambda| \approx 0.2902990904, \Omega_{1} \approx 14.08506960$ and $\Omega_{2} \approx 0.9144444033<1$. Notice that

$$
\begin{aligned}
|f(t, x)| & =\left|\frac{3}{3 t+1049}\left(x^{2}+\frac{1}{2} e^{-x^{2}}+\frac{1}{2}\right)\right| \\
& \leq\left(\frac{3}{3 t+1049}\right)\left(x^{2}+1\right):=p(t) \phi(x),
\end{aligned}
$$

which fulfills the hypothesis (H3) with $\|p\| \leq 1 / 350$ and $\phi(\mathcal{M})=\mathcal{M}^{2}+1$. Also (H4) is satisfied for $\mathcal{M} \in(0.7025154750,1.423456188)$. Hence by Theorem 3.2, the problem (3.12)(3.13) has at least one solution on $[1 / 3,5 / 3]$.

\subsection{Uniqueness result}

We shall use the Banach contraction mapping principle to prove the uniqueness of the solutions of the boundary value problem (1.1)-(1.2).

\section{Theorem 3.3 Assume that:}

(H5) There exists a positive constant $\mathcal{L}>0$ such that $|f(t, x)-f(t, y)| \leq \mathcal{L}|x-y|$, for each $t \in J$ and $x, y \in \mathbb{R}$.

Then the boundary value problem (1.1)-(1.2) has a unique solution on J, provided that

$$
\mathcal{L} \Omega_{1}+\Omega_{2}<1
$$

where the constants $\Omega_{1}, \Omega_{2}$ are defined by (3.2) and (3.3) respectively.

Proof We want to prove that the operator $\mathcal{N}: \mathcal{C} \rightarrow \mathcal{C}$ defined by (3.1) has a fixed point. This fixed point is then a solution of the problem (1.1)-(1.2). Set $\sup _{t \in J}|f(t, 0)|=\mathcal{K}<\infty$, 
$\mathcal{B}_{r}=\{x \in \mathcal{C}:\|x\| \leq r\}$ and choose $r \geq\left(\mathcal{K} \Omega_{1}\right) /\left(1-\mathcal{L} \Omega_{1}-\Omega_{2}\right)$. Then we will show that $\mathcal{N} \mathcal{B}_{r} \subset$ $\mathcal{B}_{r}$. For any $x \in \mathcal{B}_{r}$, we have

$$
\begin{aligned}
\mid(\mathcal{N} x) & (t) \mid \\
\leq & \sup _{t \in J}\left\{I^{\chi_{1}+\chi_{2} ; \psi}(|f(t, x(t))-f(t, 0)|+|f(t, 0)|)+|k| I^{\chi_{2} ; \psi}|x(t)|\right. \\
& +\frac{(\psi(t)-\psi(a))^{\gamma_{1}+\chi_{2}-1}}{\Lambda \Gamma\left(\gamma_{1}+\chi_{2}\right)}\left[\sum_{i=1}^{m}\left|\lambda_{i}\right| I^{\chi_{1}+\chi_{2}+\delta_{i} ; \psi}\left(\left|f\left(\tau_{i}, x\left(\tau_{i}\right)\right)-f\left(\tau_{i}, 0\right)\right|+\left|f\left(\tau_{i}, 0\right)\right|\right)\right. \\
& +|k| \sum_{i=1}^{m}\left|\lambda_{i}\right| I^{\chi_{2}+\delta_{i} ; \psi}\left|x\left(\tau_{i}\right)\right|+I^{\chi_{1}+\chi_{2} ; \psi}(|f(b, x(b))-f(b, 0)|+|f(b, 0)|) \\
& \left.\left.+|k| I^{\chi_{2} ; \psi}|x(b)|\right]\right\} \\
\leq & (\mathcal{L}\|x\|+\mathcal{K})\left(\frac{(\psi(b)-\psi(a))^{\chi_{1}+\chi_{2}}}{\Gamma\left(\chi_{1}+\chi_{2}+1\right)}+\frac{(\psi(b)-\psi(a))^{\gamma_{1}+\chi_{2}-1}}{|\Lambda| \Gamma\left(\gamma_{1}+\chi_{2}\right)}\right. \\
\quad & {\left.\left[\sum_{i=1}^{m}\left|\lambda_{i}\right| \frac{\left(\psi\left(\tau_{i}\right)-\psi(a)\right)^{\chi_{1}+\chi_{2}+\delta_{i}}}{\Gamma\left(\chi_{1}+\chi_{2}+\delta_{i}+1\right)}+\frac{(\psi(b)-\psi(a))^{\chi_{1}+\chi_{2}}}{\Gamma\left(\chi_{1}+\chi_{2}+1\right)}\right]\right) } \\
& +\|x\|\left(| k | \left\{\frac{(\psi(b)-\psi(a))^{\chi_{2}}}{\Gamma\left(\chi_{2}+1\right)}+\frac{(\psi(b)-\psi(a))^{\gamma_{1}+\chi_{2}-1}}{|\Lambda| \Gamma\left(\gamma_{1}+\gamma_{2}\right)}\right.\right. \\
\leq & {\left.\left.\left[\sum_{i=1}^{m}\left|\lambda_{i}\right| \frac{\left(\psi\left(\tau_{i}\right)-\psi(a)\right)^{\chi_{2}+\delta_{i}}}{\Gamma\left(\chi_{2}+\delta_{i}+1\right)}+\frac{(\psi(b)-\psi(a))^{\chi_{2}}}{\Gamma\left(\chi_{2}+1\right)}\right]\right\}\right) } \\
\leq & (\mathcal{L} r+\mathcal{K}) \Omega_{1}+r \Omega_{2}<r .
\end{aligned}
$$

Therefore $\|\mathcal{N} x\| \leq r$ which implies that $\mathcal{N} \mathcal{B}_{r} \subset \mathcal{B}_{r}$.

Now for $x, y \in \mathcal{C}$, we have

$$
\begin{aligned}
& |(\mathcal{N} x)(t)-(\mathcal{N} y)(t)| \\
& \leq\left(\frac{(\psi(b)-\psi(a))^{\chi_{1}+\chi_{2}}}{\Gamma\left(\chi_{1}+\chi_{2}+1\right)}+\frac{(\psi(b)-\psi(a))^{\gamma_{1}+\chi_{2}-1}}{|\Lambda| \Gamma\left(\gamma_{1}+\chi_{2}\right)}\right. \\
& \left.\quad \times\left[\sum_{i=1}^{m}\left|\lambda_{i}\right| \frac{\left(\psi\left(\tau_{i}\right)-\psi(a)\right)^{\chi_{1}+\chi_{2}+\delta_{i}}}{\Gamma\left(\chi_{1}+\chi_{2}+\delta_{i}+1\right)}+\frac{(\psi(b)-\psi(a))^{\chi_{1}+\chi_{2}}}{\Gamma\left(\chi_{1}+\chi_{2}+1\right)}\right]\right) \mathcal{L}\|x-y\| \\
& \quad+|k|\left(\left\{\frac{(\psi(b)-\psi(a))^{\chi_{2}}}{\Gamma\left(\chi_{2}+1\right)}+\frac{(\psi(b)-\psi(a))^{\gamma_{1}+\chi_{2}-1}}{|\Lambda| \Gamma\left(\gamma_{1}+\gamma_{2}\right)}\right.\right. \\
& \left.\left.\quad \times\left[\sum_{i=1}^{m}\left|\lambda_{i}\right| \frac{\left(\psi\left(\tau_{i}\right)-\psi(a)\right)^{\chi_{2}+\delta_{i}}}{\Gamma\left(\chi_{2}+\delta_{i}+1\right)}+\frac{(\psi(b)-\psi(a))^{\chi_{2}}}{\Gamma\left(\chi_{2}+1\right)}\right]\right\}\right)\|x-y\| \\
& =\left(\mathcal{L} \Omega_{1}+\Omega_{2}\right)\|x-y\| .
\end{aligned}
$$

Therefore $\|(\mathcal{N} x)-(\mathcal{N} y)\| \leq\left(\mathcal{L} \Omega_{1}+\Omega_{2}\right)\|x-y\|$. As $\mathcal{L} \Omega_{1}+\Omega_{2}<1 \mathcal{N}$ is a contraction. By the Banach contraction mapping principle (Lemma 2.3) we deduce that $\mathcal{N}$ has a fixed 
point, which is the unique solution of the boundary value problem (1.1)-(1.2). The proof is finished.

Example 3.3 Consider the boundary value problem of the Langevin equation with the $\psi$-HFD of the form

$$
\begin{aligned}
& \mathcal{D}^{\frac{3}{7}, \frac{2}{7} ; t^{2}+1}\left(\mathcal{D}^{\frac{5}{7}, \frac{4}{7} ; t^{2}+1}+\frac{1}{13}\right) x(t)=\frac{2}{4 t+31}\left(\frac{x^{2}(t)+2|x(t)|}{1+|x(t)|}\right)+\frac{1}{4}, \quad t \in\left[\frac{1}{4}, \frac{5}{4}\right], \\
& x\left(\frac{1}{4}\right)=0, \quad x\left(\frac{5}{4}\right)=\frac{3}{8} I^{\frac{2}{3} ; t^{2}+1} x\left(\frac{1}{2}\right)+\frac{5}{8} I^{\frac{4}{3} ; t^{2}+1} x\left(\frac{3}{4}\right)+\frac{7}{8} I^{\frac{5}{3} ; t^{2}+1} x(1) .
\end{aligned}
$$

Here $\chi_{1}=3 / 7, \chi_{2}=5 / 7, \beta_{1}=2 / 7, \beta_{2}=4 / 7, k=1 / 13, a=1 / 4, b=5 / 4, m=3, \delta_{1}=2 / 3$, $\delta_{2}=4 / 3, \delta_{3}=5 / 3, \tau_{1}=1 / 2, \tau_{2}=3 / 4, \tau_{3}=1, \lambda_{1}=3 / 8, \lambda_{2}=5 / 8, \lambda_{3}=7 / 8$ and $\psi(t)=t^{2}+1$. Note that $\chi_{1}+\chi_{2}=8 / 7 \in(1,2]$.

With these values we found $\gamma_{1}=29 / 49, \gamma_{2}=43 / 49,|\Lambda| \approx 0.6572399879, \Omega_{1} \approx$ 4.725942806 and $\Omega_{2} \approx 0.3825052846<1$ and $\mathcal{L}=1 / 8$ as

$$
|f(t, x)-f(t, y)| \leq \frac{1}{8}|x-y|, \quad \text { for any } x, y \in \mathbb{R}
$$

Since $\mathcal{L} \Omega_{1}+\Omega_{2} \approx 0.9732481354<1$, by Theorem 3.3, the problem (3.15)-(3.16) has a unique solution on $[1 / 4,5 / 4]$.

\section{Acknowledgements}

Not applicable.

\section{Funding}

This research was funded by King Mongkut's University of Technology North Bangkok. Contract no. KMUTNB-61-KNOW-032.

\section{Availability of data and materials}

Data sharing not applicable to this article as no data sets were generated or analyzed during the current study.

\section{Competing interests}

The authors declare that they have no competing interests.

\section{Authors' contributions}

All authors contributed equally to this work. All authors read and approved the final manuscript.

\section{Author details}

'Thai-German Pre-engineering School, College of Industrial Technology, King Mongkut's University of Technology North Bangkok, Bangkok, 10800, Thailand. '2Department of Mathematics, University of loannina, 45110 loannina, Greece. ${ }^{3}$ Nonlinear Analysis and Applied Mathematics (NAAM)-Research Group, Department of Mathematics, Faculty of Science, King Abdulaziz University, P.O. Box 80203, Jeddah 21589, Saudi Arabia. ${ }^{4}$ Department of Mathematics, PSG College of Arts \& Science, Coimbatore, 641014, India. ${ }^{5}$ Intelligent and Nonlinear Dynamic Innovations, Department of Mathematics, Faculty of Applied Science, King Mongkut's University of Technology North Bangkok, Bangkok, 10800, Thailand.

\section{Publisher's Note}

Springer Nature remains neutral with regard to jurisdictional claims in published maps and institutional affiliations.

Received: 28 December 2020 Accepted: 14 March 2021 Published online: 25 March 2021

\section{References}

1. Diethelm, K.: The Analysis of Fractional Differential Equations. Lecture Notes in Mathematics. Springer, New York (2010)

2. Kilbas, A.A., Srivastava, H.M., Trujillo, J.J.: Theory and Applications of the Fractional Differential Equations. North-Holland Mathematics Studies, vol. 204 (2006)

3. Lakshmikantham, V., Leela, S., Devi, J.V.: Theory of Fractional Dynamic Systems. Cambridge Scientific Publishers (2009)

4. Miller, K.S., Ross, B.: An Introduction to the Fractional Calculus and Differential Equations. Wiley, New York (1993) 
5. Podlubny, I.: Fractional Differential Equations. Academic Press, New York (1999)

6. Samko, S.G., Kilbas, A.A., Marichev, O.I.: Fractional Integrals and Derivatives. Gordon \& Breach, Yverdon (1993)

7. Zhou, Y.: Basic Theory of Fractional Differential Equations. World Scientific, Singapore (2014)

8. Feckan, M., Wang, J.R.: Periodic impulsive fractional differential equations. Adv. Nonlinear Anal. 8(1), 482-496 (2019)

9. Ahmad, B., Alsaedi, A., Ntouyas, S.K., Tariboon, J.: Hadamard-Type Fractional Differential Equations, Inclusions and Inequalities. Springer, Cham (2017)

10. Hilfer, R.: Applications of Fractional Calculus in Physics. World Scientific, Singapore (2000)

11. da Vanterler, J., Sousa, C., Capelas de Oliveira, E.: On the $\psi$-Hilfer fractional derivative. Commun. Nonlinear Sci. Numer. Simul. 60, 72-91 (2018)

12. Capelas de Oliveira, E., da Vanterler, J., Sousa, C.: Ulam-Hyers-Rassias stability for a class of fractional integro-differential equations. Results Math. 73(3), 1-16 (2018)

13. da Vanterler, J., Sousa, C., Benchohra, M., N'Guérékata, G.M.: Attractivity for differential equations of fractional order and $\psi$-Hilfer type. Fract. Calc. Appl. Anal. 23, 1188-1207 (2020)

14. Boudjerida, A., Seba, D., N'Guérékata, G.M.: Controllability of coupled systems for impulsive $\phi$-Hilfer fractional integro-differential inclusions. Appl. Anal. (2020). https://doi.org/10.1080/00036811.2020.1742884

15. da Vanterler, J., Sousa, C., Jarad, F., Abdeljawad, T.: Existence of mild solutions to Hilfer fractional evolution equations in Banach space. Ann. Funct. Anal. 12, 12 (2021)

16. Benchohra, M., Bouriah, S., Henderson, J.: Ulam stability for nonlocal differential equations involving the Hilfer-Katugampola fractional derivative. Afr. Math. (2021). https://doi.org/10.1007/s13370-020-00864-4

17. da Vanterler, J., Sousa, C., Kucche, K.D., Capelas de Oliveira, E.: Stability of $\psi$-Hilfer impulsive fractional differential equations. Appl. Math. Lett. 88, 73-80 (2019)

18. da Vanterler, J., Sousa, C., Capelas de Oliveira, E.: On the Ulam-Hyers-Rassias stability for nonlinear fractional differential equations using the $\psi$-Hilfer operator. J. Fixed Point Theory Appl. 20(3), 1-21 (2018)

19. Asawasamrit, S., Kijjathanakorn, A., Ntouyas, S.K., Tariboon, J.: Nonlocal boundary value problems for Hilfer fractional differential equations. Bull. Korean Math. Soc. 55(6), 1639-1657 (2018)

20. Sudsutad, W., Ntouyas, S.K., Tariboon, J.: Systems of fractional Langevin equations of Riemann-Liouville and Hadamard types. Adv. Differ. Equ. 2015, 235 (2015)

21. Harikrishnan, S., Shah, K., Kanagarajan, K.: Study of a boundary value problem for fractional order $\psi$-Hilfer fractional derivative. Arab. J. Math. 2019, 1-8 (2019)

22. Saengthong, W., Thailert, E., Ntouyas, S.K.: Existence and uniqueness of solutions for system of Hilfer-Hadamard sequential fractional differential equations with two point boundary conditions. Adv. Differ. Equ. 2019, 525 (2019)

23. Ahmed, H.M., El-Borai, M.M.: Hilfer fractional stochastic integro-differential equations. Appl. Math. Comput. 331, 182-189 (2018)

24. Gou, H., Li, Y.: Upper and lower solution method for Hilfer fractional evolution equations with nonlocal conditions. Bound. Value Probl. 2019, 187 (2019)

25. Langevin, P.: On the theory of Brownian motion. Compt. Rendus 146, 530-533 (1908)

26. Mazo, R.M.: Brownian Motion: Fluctuations, Dynamics, and Applications. Oxford University Press, Oxford (2002)

27. Wax, N.: Selected Papers on Noise and Stochastic Processes. Dover, New York (1954)

28. Zwanzig, R.: Nonequilibrium Statistical Mechanics. Oxford University Press, Oxford (2001)

29. West, B.J., Latka, M.: Fractional Langevin model of gait variability. J. NeuroEng. Rehabil. 2(1), 1-24 (2005)

30. Picozzi, S., West, B.J.: Fractional Langevin model of memory in financial markets. Phys. Rev. E 66(4), 46 (2002)

31. Kobelev, V., Romanov, E.: Fractional Langevin equation to describe anomalous diffusion. Prog. Theor. Phys. Suppl. 139, 470-476 (2000)

32. Harikrishnan, S., Kanagarajan, K., Elsayed, E.M.: Existence and stability results for Langevin equations with Hilfer fractional derivative. Res. Fixed Point Theory Appl. 2018, Article ID 20183 (2018)

33. Lim, S.C., Li, M., Teo, L.P.: Langevin equation with two fractional orders. Mod. Phys. Lett. A 372(42), 6309-6320 (2008)

34. Lizana, L., Ambjörnsson, T., Taloni, A., Barkai, E., Lomholt, M.A.: Foundation of fractional Langevin equation: harmonization of a many-body problem. Phys. Rev. E 81(5), 051118 (2010)

35. Yukunthorn, W., Ntouyas, S.K., Tariboon, J.: Nonlinear fractional Caputo-Langevin equation with nonlocal Riemann-Liouville fractional integral conditions. Adv. Differ. Equ. 2014, 315 (2014)

36. Wongcharoen, A., Ahmad, B., Ntouyas, S.K., Tariboon, J.: Three-point boundary value problems for Langevin equation with Hilfer fractional derivative. Adv. Math. Phys. 2020, Article ID 9606428 (2020)

37. Deimling, K.: Nonlinear Functional Analysis. Springer, New York (1985)

38. Krasnosel'skiï, M.A.: Two remarks on the method of successive approximations. Usp. Mat. Nauk 10, 123-127 (1995)

39. Granas, A., Dugundji, J.: Fixed Point Theory. Springer, New York (2003) 